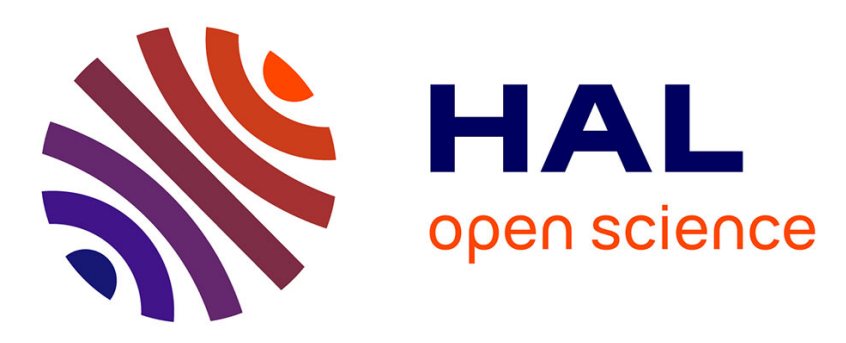

\title{
Towards adapting current 3DTV for an improved 3D experience
}

Laurent Blonde, Didier Doyen, Cédric Thébault, Quan Huynh-Thu, Daniel Stoenescu, Emmanuel Daniel, Jean-Louis de Bougrenet de La Tocnaye, Samir Bentahar

\section{To cite this version:}

Laurent Blonde, Didier Doyen, Cédric Thébault, Quan Huynh-Thu, Daniel Stoenescu, et al.. Towards adapting current 3DTV for an improved 3D experience. Speech Communication, 2012, $10.1117 / 12.906985$. hal-00724683

\section{HAL Id: hal-00724683 \\ https://hal.science/hal-00724683}

Submitted on 14 Jun 2021

HAL is a multi-disciplinary open access archive for the deposit and dissemination of scientific research documents, whether they are published or not. The documents may come from teaching and research institutions in France or abroad, or from public or private research centers.
L'archive ouverte pluridisciplinaire HAL, est destinée au dépôt et à la diffusion de documents scientifiques de niveau recherche, publiés ou non, émanant des établissements d'enseignement et de recherche français ou étrangers, des laboratoires publics ou privés.

\section{(c)(1)}

Distributed under a Creative Commons Attribution| 4.0 International License 


\title{
Towards adapting current 3DTV for an improved 3D experience
}

\author{
L. Blondé ${ }^{1}$, D. Doyen ${ }^{1}$, C. Thébault ${ }^{1}$, Q. Huynh Thu ${ }^{1}$, \\ D. Stoenescu ${ }^{2}$, E. Daniel ${ }^{2}$, J.L. de Bougrenet de la Tocnaye ${ }^{2}$, \\ S. Bentahar ${ }^{3}$ \\ ${ }^{1}$ Technicolor, 1 av. de Belle-Fontaine, 35576 Cesson-Sévigné, France \\ ${ }^{2}$ Télécom Bretagne, Technopôle Brest-Iroise, 29238 Brest, France \\ ${ }^{3}$ E3S, Technopôle Brest-Iroise, 29280 Plouzané, France
}

\begin{abstract}
Recent upgrades of HDTV into 3DTV resulted in impairments in displaying stereo contents. One of the most critical flaws is probably crosstalk and the resultant ghosting effect impairing the 3D experience. The purpose of this study is to identify the primary source of crosstalk, throughout the full image generation and viewing chain, for a selection of 3D displays: Liquid Crystal Display (LCD) and Plasma Display Panel (PDP) combined with different active glasses technologies. Time measurements have been carried out on various display panels and shutter glasses technologies. For each technology, the crosstalk is a complex combination of several factors depending on display panels, shutter glasses and their synchronization, and ghost busting. The study tried to discriminate the main sources of crosstalk in each case, and to simulate the effect of various display panels or shutter glasses performance optimizations. Analysis and conclusions vary depending on the display technology. For LCD, light leakage at the panel level appears the first cause of crosstalk, and, in a second step, optimization of the shutter glasses. For PDP the use of more adapted shutter glasses can mitigate color distortion effects.
\end{abstract}

Keywords: 3DTV, 3D-Cinema, Stereoscopy, Shutter Glasses.

\section{INTRODUCTION}

In the main context of 3DTV or 3D games developments, it is crucial to analyze and well understand the technical characteristics of 3D displays with the goal to identify solutions for an improved 3D experience. Recent upgrades of HDTV into 3DTV have resulted in some impairment in displaying stereo contents. One of the most critical imperfections is probably crosstalk (the leakage of one view into the other one). The resulting ghosting effect often strongly impairs the quality of the 3D experience. This technical contribution aims at studying the crosstalk effect in the context of active glasses 3D displays and suggesting some improvements. Both LCD and PDP will be studied with approaches focusing on the main limitation of each technology.

Compared to 2D-HDTV, 3D crosstalk is markedly new for TV viewers as a source of discomfort and fatigue. For the time being, they have to accept it until 3D technologies should be significantly improved. If not, the alternative could be a rejection of $3 \mathrm{DTV}$ as a whole, with the risk to impinge the 3D industry perspectives. The purpose of this study is to identify the primary source of crosstalk, throughout the full image generation and viewing chain, for a selection of commercial active 3D displays (LCD and PDP) combined with different glasses technologies (often overly considered as responsible for crosstalk). Both the glasses responses and the screen emitted light signals were examined and the contribution of each element to crosstalk was analyzed from measurements and simulations.

Although far from being exhaustive and based on currently commercially available displays and glasses, the study is indicative of trends to follow towards quality improvement. More specifically, it is shown that innovative glasses technologies require improved characteristics from the display panel to express their best performances.

For an active 3D display system, an analysis should focus on one hand on the display panel (including lighting technology, image processing and compensation) and on the other hand on the shutter glasses and their triggering as 
reported in [1][2][3]. Measuring the panel emitted light signal, and its temporal evolution, is a first step. With this knowledge and measurements carried out on the shutter glasses, crosstalk can be studied, both in its light leakage and temporal aspects, even in presence of ghost busting processing.

For this study, time measurements have been carried out on various display panels and shutter glasses technologies. Display panels measured include a sampling of 3DTV screens commercially available in 2010 and 2011, shutter glasses provided with the displays as well as prototypes with optimized characteristics. Several panels were tested: a representative PDP and two LCDs. In contrast to PDP, LCDs are based on different liquid crystal technologies and have different backlight management strategies. On this basis, we carried out evaluations and simulations to estimate panel and shutter glasses performance and assess how their combination could be optimized to mitigate the crosstalk effect.

LCD panels exhibit liquid crystal response time and backlight synchronization issues, as well as some artifacts due to LC overdriving techniques. PDPs suffer from phosphor lag and need a clever sub-fields management strategy to reduce defects. All types of panels need to adapt the shutter glasses synchronization with respect to their technology (rarely done) and can be impacted by over-compensation or inaccuracy of ghost busting techniques used by most manufacturers to overcome some current ghosting issues. In practice, ghosting compensation could not be decoupled from the physical aspects of crosstalk, so for LCDs only white leakage on a black background was used as evaluation criteria, in a situation where theoretically anti-ghosting does not operate. For PDP used in 3D, the number of subfields is reduced and their order is reversed to mitigate the phosphor leakage effect, according to the increase in frame rate.

\section{EVALUATION OF LIQUID CRYSTAL DISPLAY (LCD) PANEL BASED 3D DISPLAYS}

Two consumer 3D LCD displays, from two different manufacturers, were measured in this study in their standard settings, except when mentioned. Not to disclose manufacturers' names these two displays are referred to as LCD1 and LCD2 in this document. They were commercially available during spring 2010 and spring 2011 respectively.

LCD1 and LCD2 3DTV models have different backlight, scanning and shutter management strategies. From display and shutter glasses measurements we could estimate these strategies. It appears that they follow the temporal evolution as presented in Figure 1 and Figure 2 respectively. LCD1 has half-period opened states for the left and right eye shutter and separation between left and right signal is realized by a black signal insert and a synchronized black period for the backlight pulses. LCD2 has narrower shutter opened states, covering approximately one quarter of the 1/60s period, and during this period the bottom half and then the top half of the screen are backlit. Line addressing is done during the two quarter periods when the left and right shutters are simultaneously in their blocking state.

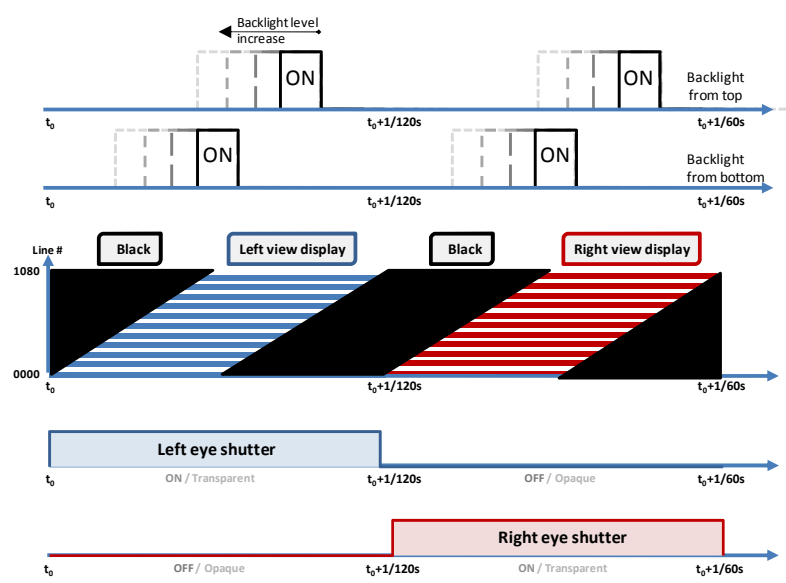

Figure 1. LCD1 backlight, scanning and shutter management

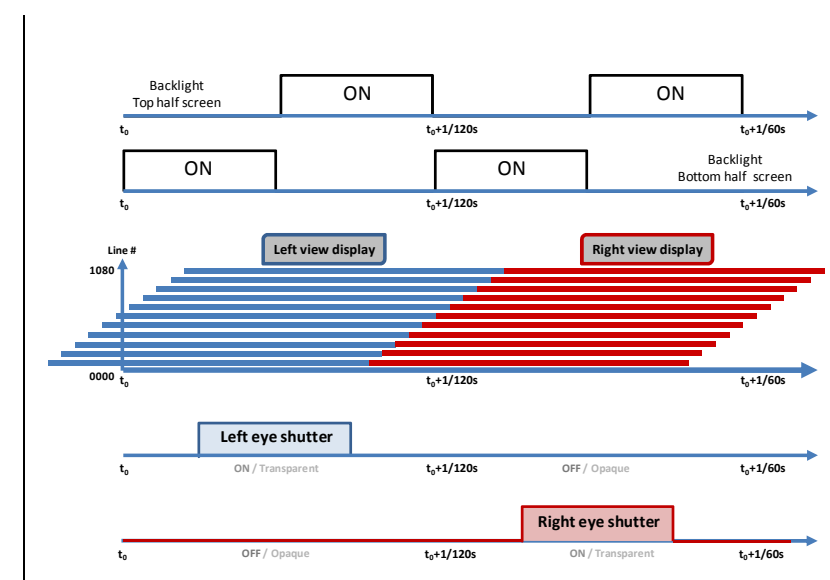

Figure 2. LCD2 backlight, scanning and shutter management 


\subsection{Temporal Measurement}

Literature proposes different formulas for crosstalk evaluation. In the present discussion, diaphoty photopic contrast is used: $C=L^{L L} / L^{L R}$ with $L^{L L}$ being luminance measured in the left channel, with signal emitted for the left channel only and $L^{L R}$ being luminance measured in the right channel, with signal emitted for the left channel only.

Time measurements have been done on the studied active LCD displays where the sequentially displayed images and the shutter glasses contribute to the temporal separation and presentation of the stereo pairs. Time measurement can identify chronograms of the light emitted by the screen and how they trigger the shutter glasses switching states. As the goal is to perform simulations of various scaling and phasing options for the shutter responses, temporal measurements were done separately for the displays and for the shutter glasses, using as common basis the 3D display synchronization signal.

These measurements used a Fabry cell assembly setup as presented in [4], controlling measurement conditions with care. Based on the combination of a photosensitive cell, a lens and a pupillar aperture, this optical arrangement allows an accurate control of the measured field of view (see Figure 3).

\subsubsection{Measuring Glasses only}

The glasses measurement setup consists in placing the photosensor cells at the eye position behind the glasses, and illuminating them with a stabilized DC current tungsten lamp equipped with a condenser and UV/IR filters (see Figure 4). Potentially a linear polarizer is used if not present in the glasses design (when polarized light output from the display is assumed). In the chosen configuration the glasses synchronization sensor is lit directly by the 3DTV IR photodiode, while image light output from the 3DTV is blocked with a physical screen. Some example of glasses measurements are shown on Figure 15.

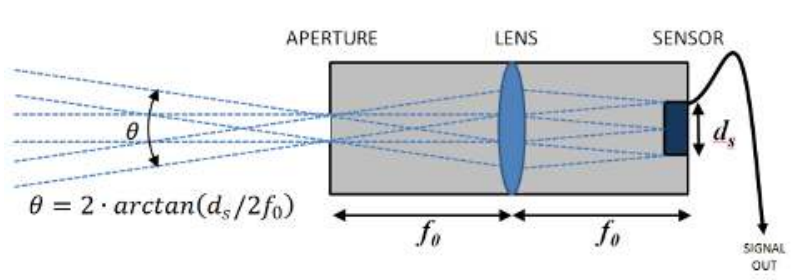

Figure 3. Fabry cell assembly optical arrangement

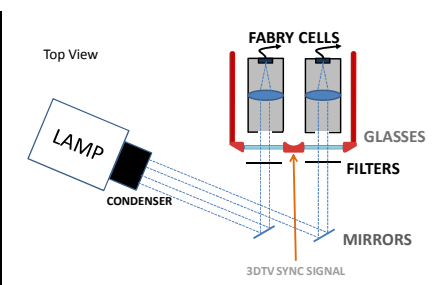

Figure 4. Setup for glasses measurement.

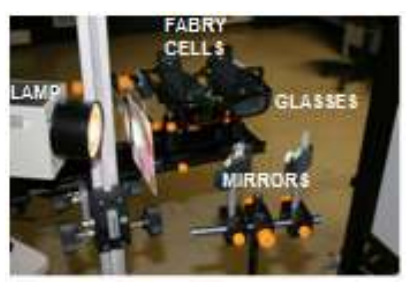

\subsubsection{Measuring Display only}

Although it is not a crosstalk measurement as such, measuring the display without glasses is a very informative experiment. Indeed, the temporal variations of the signals can be really instructive about the display technology and the light management strategy selected by the display manufacturer. For example, in the case of LCD displays, backlight management, screen scanning and addressing can be analyzed.

Measuring the display with a temporal method requires the same setup as described in the 'Glasses only' sub-section. In this case, the 3DTV signal is observed directly with the Fabry cell assembly and various vertical positions reveal the temporal scanning, from top to bottom of the screen.

Figure 5 and Figure 6 present measurements on LCD1 in specific conditions.

- Figure 5: different grey levels were applied in 3D mode, with same level applied on both views. This is a configuration where no crosstalk is generated as the two views (left view before $0.01 \mathrm{~s}$, right view after $0.01 \mathrm{~s}$ ) are measured identical. The tripartite structure of the responses can be explained by the backlight management (see next comment). 
- Figure 6: an image with a white left view $(L=255)$ and a black right view $(R=0)$ has been measured for different backlight levels (from $\max =20$ to $\min =0$ on the manufacturers GUI scale). Low levels show that the backlight is composed of two pulses for each of the $\mathrm{L}$ views, with an echo on the R views. Depending on the backlight level, the two pulses are separated (low levels) or overlap (high levels) creating then the tripartite structure.

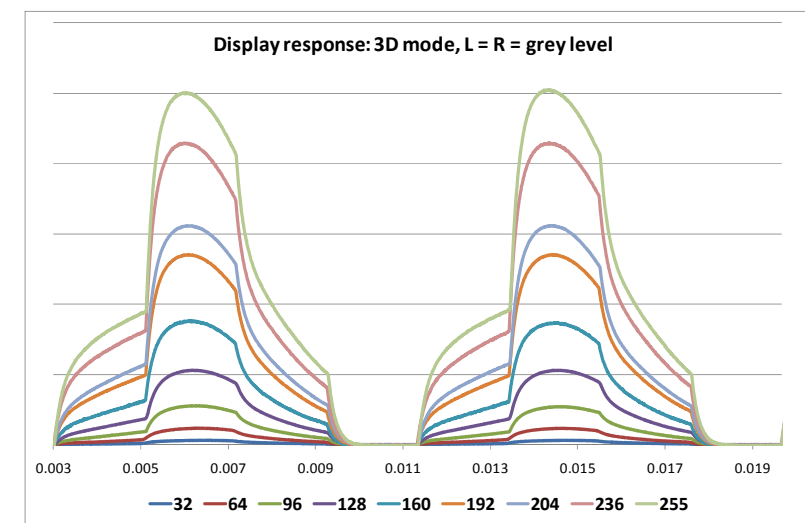

Figure 5. LCD1 display direct measurement, left and right views set to grey for different levels

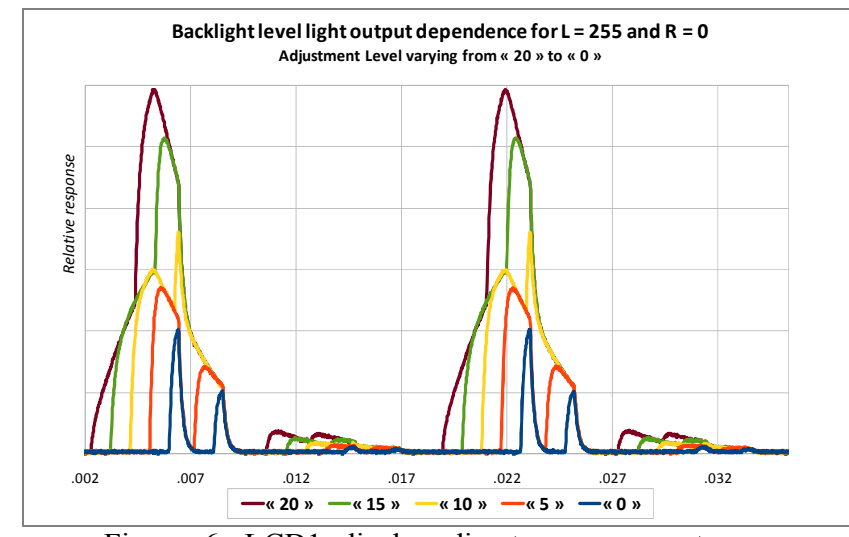

Figure 6. LCD1 display direct measurement, adjusting backlight level from max to min

On Figure 6, an important light echo is visible between $0.010 \mathrm{~s}$ and $0.018 \mathrm{~s}$, during a right view half-period, when no light is expected for a $R$ signal set to $R=0$.

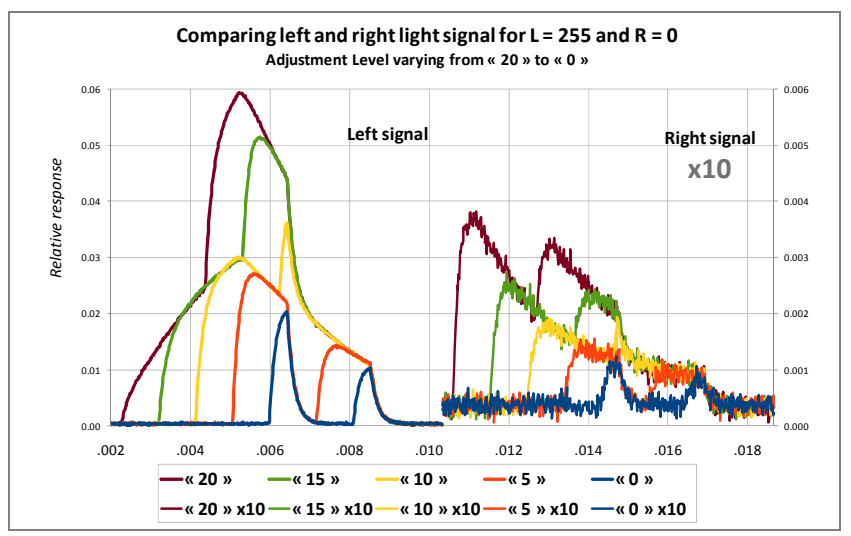

Figure 7. Same light signal as Figure 6, with right halfperiod signal amplified $(\mathrm{x} 10)$.

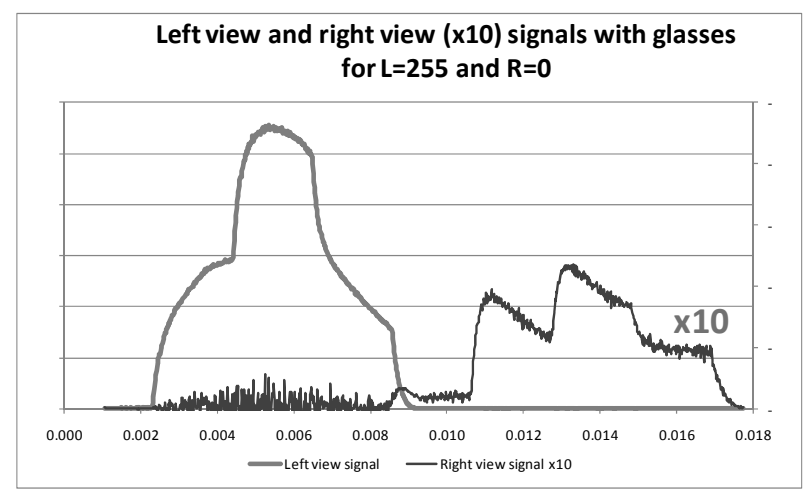

Figure 8. Simultaneous measurement of left view and right view signal through LCD1 shutter glasses.

Figure 7 presents the same light signal as Figure 6 but with a stretched timeline and with the second half-period signal (right view half-period) amplified by a factor ten. The structure of the light echo is more visible, and can be associated with the two pulses forming the signal of the first half period for each backlight level. How light echo is generated is not know by the authors, but clearly, as this light is emitted when the right shutter is fully open, it directly contributes to the diaphoty photopic contrast decrease and thus to crosstalk.

On Figure 8 a full 3D period is shown (spanning $1 / 60 \mathrm{~s}=16.66 \mathrm{~ms}$ ), with measurements for both the left and right eye of LCD1 shutter glasses. The 3D display digital signal is identical to Figure 6 with a white left view $(L=255)$ and a black 
right view $(R=0)$. As right image level is $R=0$, right view signal is expected to be totally black. The measured light level is thus unintended signal generating crosstalk.

The measurement curves of Figure 8 are composed of two half-periods with the following characteristics:

- During the first half-period:

- Light is emitted to create the left view signal corresponding to digital level $L=255$.

$\circ$ The left eye shutter is opened, transmitting the intended light signal to the left eye.

- The right eye shutter is blocking light emitted for the left eye, giving some residual light signal seen by the right eye: this shutter leakage is participating to crosstalk

- During the second half-period:

- A light echo is emitted while no light should be emitted corresponding to digital level $R=0$.

$\circ$ The left eye shutter is blocking light, attenuating this light echo. Almost no light reaches the left eye.

$\circ$ The right eye shutter is opened, transmitting the unintended light echo to the right eye. This display leakage is participating to crosstalk.

On the measurement of Figure 8, one can notice the strong dominance of the display leakage of the second half-period compared to the shutter leakage of the first half-period. Furthermore, the second half period is intended black so the second half period leakage generates a significant crosstalk contrast.

Figure 10 and Figure 11 present measurements on the second studied Liquid Crystal Display, LCD2. As presented on Figure 2, LCD2 has a significantly different backlight, scanning and shutter management strategy compared to LCD1.

Figure 10 helps understanding this strategy showing display measurements for five positions from top to bottom of the screen and showing the LCD2 shutter responses on the same timescale. As before the 3D digital levels are $L=255$ and $R=0$. Figure 10 and complementary measurements allowed identifying four quarter-periods in the display signal, alternating between left and right views and between top ( $\mathrm{C} 1$ and $\mathrm{C} 2$ curves) and bottom ( $\mathrm{C} 4$ and $\mathrm{C} 5$ curves) halves of the screen ${ }^{1}$ as summarized in Figure 9. The right and left shutters open with a time shift adequate to integrate the light emitted by the top and bottom halves of the screen for each respective views.

\begin{tabular}{|c|c|c|c|c|}
\hline & Quarter-period 1 & Quarter-period 2 & Quarter-period 3 & Quarter-period 4 \\
\hline Displayed View & \multicolumn{2}{|c|}{ Right view } & \multicolumn{2}{|c|}{ Left view } \\
\hline Backlight ON & Bottom $(\mathrm{CA}, \mathrm{C5})$ & Top $(\mathrm{C1}, \mathrm{C2})$ & Bottom $(c 4,55)$ & Top (c1, c2) \\
\hline Shutter & Right e & tter ON & Left ey & er ON \\
\hline
\end{tabular}

Figure 9. LCD2 backlight, scanning and shutter periods

Figure 11 presents a full 1/60s period measurement of the LCD2 display through the right and left eye of the associated shutter glasses, with still digital levels set to $L=255$ and $R=0$. The right side signal has been enlarged by a factor ten on the diagram and represents an unintended right view light echo, when the right eye should ideally receive no light. This light echo generates a significant crosstalk contrast, due to light emission by the display during a time period when the right shutter is on. Display leakage strongly affects image quality for this screen LCD2.

\footnotetext{
${ }^{1}$ Measurement position C3, located at the center of the screen, measured light emitted during both the top and bottom quarter-periods.
} 


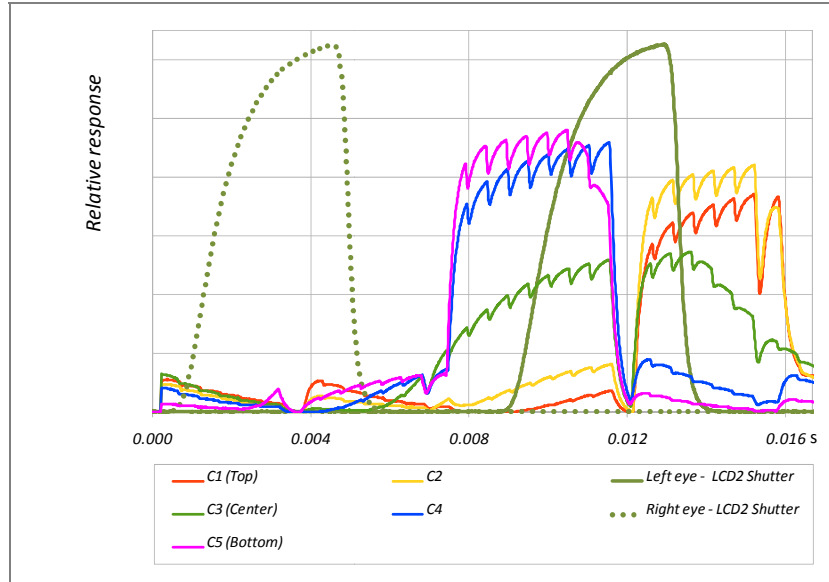

Figure 10. LCD2 display chronograms for positions $\mathrm{C} 1, \mathrm{C} 2$, C3, C4, C5 and for LCD2 original shutter technology (both shutter state, e.g. right and left eyes).
Left view and right view (x10) signals with glasses for $\mathrm{L}=\mathbf{2 5 5}$ and $\mathrm{R}=\mathbf{0}$

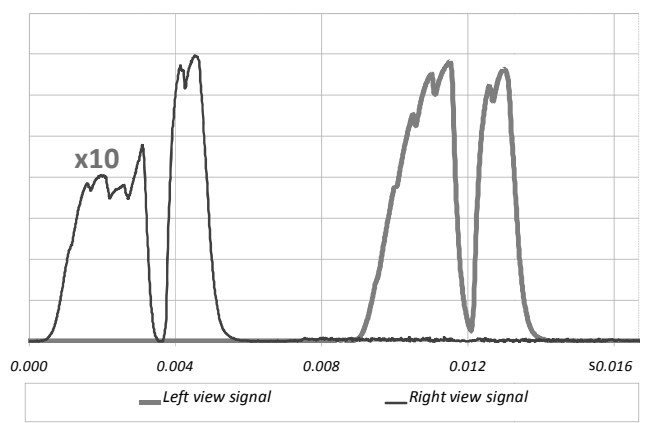

Figure 11. Simultaneous measurement of right view (x10) and left view signal through LCD2 shutter glasses.

\subsection{Simulation Principles}

The previous section identified two causes of leakage during a right view half-period:

- Display leakage: screen is still emitting light from the left view half period while the right eye shutter is open.

- Shutter leakage: the left view image light is not blocked enough by the right eye shutter.

The following study focuses on Display leakage and on display/shutter temporal synchronization aspects, evaluating by simulations how the temporal adjustment of the display signal could decrease crosstalk on a display with such leakage. The goal of the following simulations is thus to test if scaling or phasing different display signals can provide a gain in term of diaphoty photopic contrast when visualizing 3D displays. Different measurements were made and combined to create simulated display signals with several values of phase and scaling. Different shutter responses where then applied. Simulation has been carried out only on LCD1 display and results are presented in the following sections. Because display leakage is as well significant for LCD2 it is anticipated such simulation would give similar results.

\subsubsection{Display Measurement Pattern}

The 3D pattern used for crosstalk measurement has one of the two views (e.g. the right view) black (digital count set to 0 ) while the other view is composed of 25 white spots (digital count set to 255). Letters from A to E and numbers from 1 to 5 identify the spots positions as shown on Figure 12 and Figure 13.

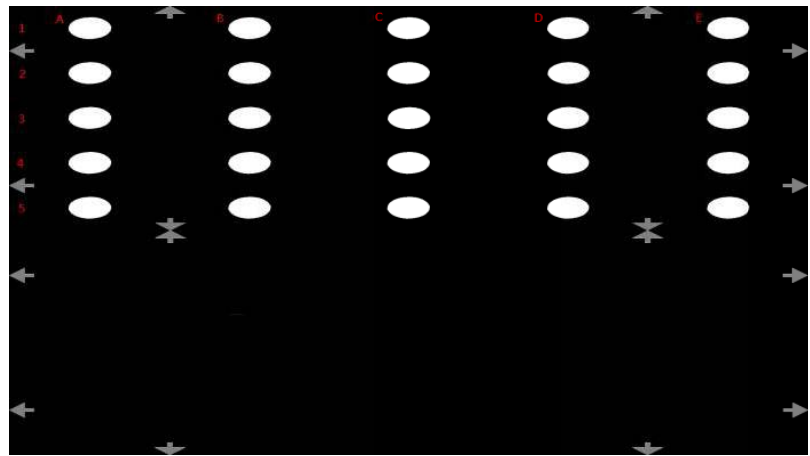

Figure 12. 3D pattern used, top-bottom format Spots level , Background level

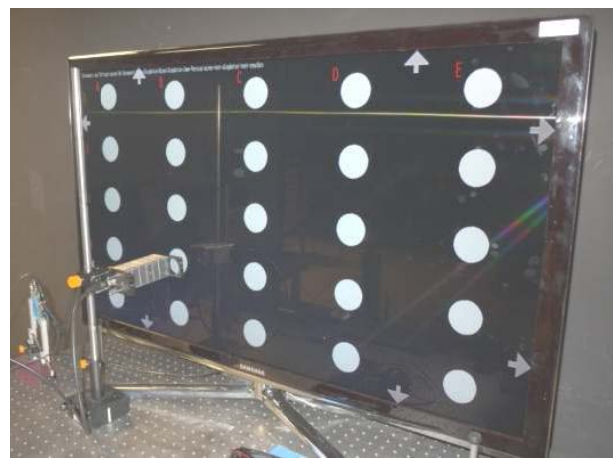

Figure 13. Fabry cell measuring spot B4 
For the purpose of the simulations median spots $\mathrm{C} 1$ to $\mathrm{C} 5$ have been measured temporally for each display. Why they were chosen and how they are combined is explained in the following sections.

\subsubsection{Simulation input data}

The current simulation is based on five chronograms for positions $\mathrm{C} 1$ to $\mathrm{C} 5$. Chronograms are similar for other columns A, B, D or E. These C1 to C5 chronograms, represented with dashed curves on Figure 14, have been measured for a maximal backlight level and, this way, present a maximal temporal extension. These $\mathrm{C} 1$ to $\mathrm{C} 5$ measurement curves change in shape and show a temporal shift depending on their position on screen (row 1 to 5). This corresponds to the bottom to top backlight scanning characteristics in the display.

Three shutter response curves are as well used. They are shown on Figure 15 for both eyes (e.g. left or right eye of shutter glasses opened). The 3 pairs of curves correspond to 3 shutter technologies (plain curves for left eye, dotted curves for right eye):

- The Original Shutter glasses provided with the LCD1 3D display (TN type).

- A Twisted Nematic shutter technology available to the authors

- A Thin Nematic shutter technology available to the authors

- In the simulation, input data are, on one hand, the $\mathrm{C} 1$ to $\mathrm{C} 5$ light measurement signals (see dotted curves of Figure 14) combined by computing their average $A v g C$. This combination $A v g C$, also named synthetic display signal hereafter, represents an actual signal of the display when observing simultaneously the five spots (see double line curve, Figure 15). This synthetic signal has the advantage to express a maximal temporal extent, relevant for the following study. Smaller temporal extends would allow more latitude in shutter response scaling and phasing, not representative of a worst case.

- On a second hand, input data include the time responses of 3 shutter technologies, measured with a stabilized DC lamp: original glasses shutter as available with the display and shutters build from two shutter technologies available to the authors: Twisted Nematic and Thin Nematic. Measured shutter responses are shown on Figure 15 for LCD1 triggering.

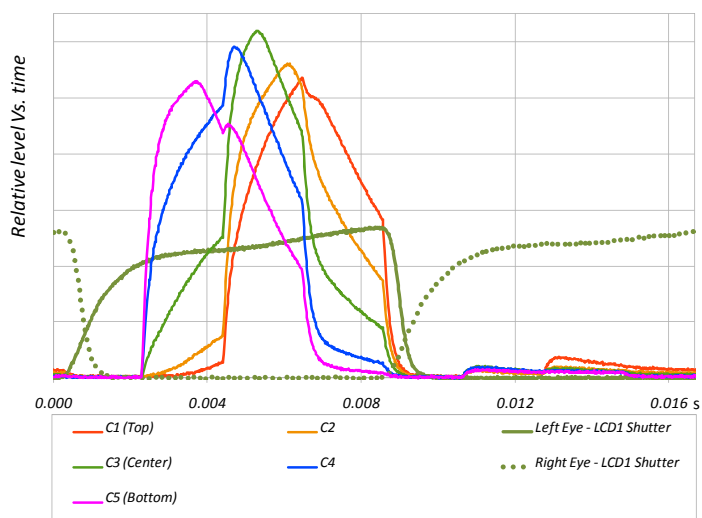

Figure 14. LCD1 display chronograms for positions $\mathrm{C} 1, \mathrm{C} 2, \mathrm{C} 3, \mathrm{C} 4, \mathrm{C} 5$ and for LCD1 original shutter technology (both shutter state, e.g. left and right eyes).

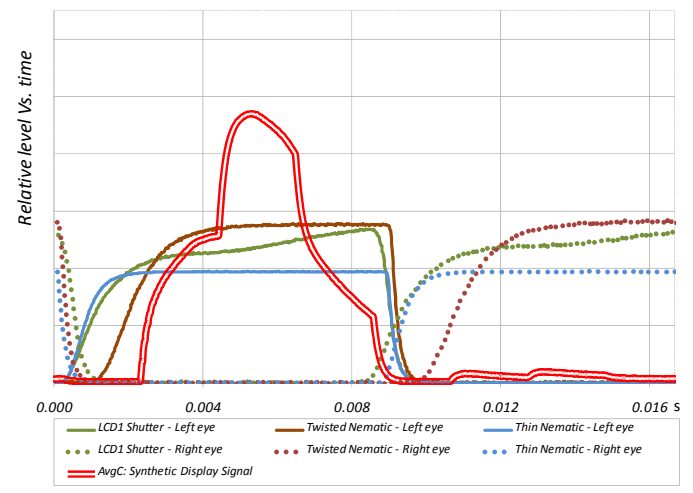

Figure 15. AvgC: Synthetic Display Signal for LCD1 display superimposed with 3 shutter technologies responses (left channels (plain lines) and right channels (dotted lines)). 
As the simulation focuses on temporal parameters, and as the black level measured with Fabry cells may present an electronic bias, the choice has been made to normalize the black level of the shutter response curves so that the intrinsic contrast becomes 1000:1 for each of the three shutter technologies measurements.

By intrinsic contrast of an adjusted shutter response we mean:

$$
C_{\text {Sht }}=\int_{t}^{t+\frac{1}{\mathrm{f}_{\text {Sht }}}} R_{\text {Sht }}(t) \cdot d t / \int_{t+\frac{1}{\mathrm{f}_{\text {Sht }}}}^{t+\frac{2}{\mathrm{f}_{\text {Sht }}}} R_{\text {Sht }}(t) \cdot d t
$$

where $R_{S h t}(t)$ is the shutter response (transparency), the shutter being in its open state in the $\left[t, t+\frac{1}{\mathrm{f}_{\text {Sht }}}\right]$ integration interval and in its close state in the $\left[t+\frac{1}{\mathrm{f}_{\mathrm{Sht}}}, t+\frac{2}{\mathrm{f}_{\mathrm{Sht}}}\right]$ integration interval when $\mathrm{f}_{\mathrm{Sht}}$ is the shutter frequency.

With these normalized shutter response curves, the comparison of the technologies will thus concentrate on the time characteristics exhibited by each technology.

\subsubsection{Simulated parameters}

In the simulation, the simulated parameters (i.e. the values compared between different configurations) is the diaphoty photopic contrast: $C=L^{L L} / L^{L R}$ with $L^{L L}$ being luminance measured in the left channel, with signal emitted for the left channel only and $L^{L R}$ being luminance measured in the right channel, with signal emitted for the left channel only.

$C=L^{L L} / L^{L R}$ is computed in the following way:

For each shutter technology (Sht)

- Compute the 'right eye' signal integral between $t$ and $t+1 / 60 s: L^{L R}=\int_{t}^{t+\frac{1}{60}} R_{\text {Sht }}^{R i g h t}(t) \cdot S_{\text {lum }}(t) \cdot d t$

- Compute the 'left eye' signal integral between $t$ and $t+1 / 60 s: L^{L L}=\int_{t}^{t+\frac{1}{60}} R_{S h t}^{L e f t}(t) \cdot S_{\text {lum }}(t) \cdot d t$

- Compute the diaphoty photopic contrast: $C=L^{L L} / L^{L R}$

where $R_{S h t}^{\text {Left }}$ and $R_{\text {Sht }}^{\text {Right }}$ are the left-eye and right-eye shutter responses (transmission) for each shutter technology. $S_{\text {lum }}(t)$ is the (real or simulated) light signal emitted by the display.

\subsubsection{Simulation process}

For the simulations $S_{\text {lum }}$ light signal is computed from $A v g C$, the synthetic display signal defined above combining $\mathrm{C} 1$ to $\mathrm{C} 5$ spots light emission.

The simulation consisted in varying the delay (phase) or the width (scale) of the $S_{\text {lum }}=\operatorname{Avg} C$ light pulses and to compute the diaphoty photopic contrast $C=L^{L L} / L^{L R}$ for different shutter glasses responses. The goal was to evaluate if faster shutter glasses would decrease diaphoty photopic contrast, with the assumption that the use of a wider shutter response, with shorter rise and fall times will increase the contrast between the intended and unintended light signals.

The AvgC signal has been transformed:

- With a variable scale factor, using the fall front as fixed reference point (scale factors 1.0, 1.1, 1.2, 1.3)

- With a variable delay (phases $-10^{\circ}, 0^{\circ}, 10^{\circ}, 20^{\circ}, 30^{\circ}, 40^{\circ}$, with $360^{\circ}=1 / 60 \mathrm{~s}$ )

Each half period (screen $\mathrm{ON}$ and screen OFF) are transformed the same way.

At their maximum these transforms bring the light signal to the maximum extend of the shutter responses in their ON state. 
Figure 16 and Figure 17 present the scale-only and delay-only transformed signals for LCD1 display.

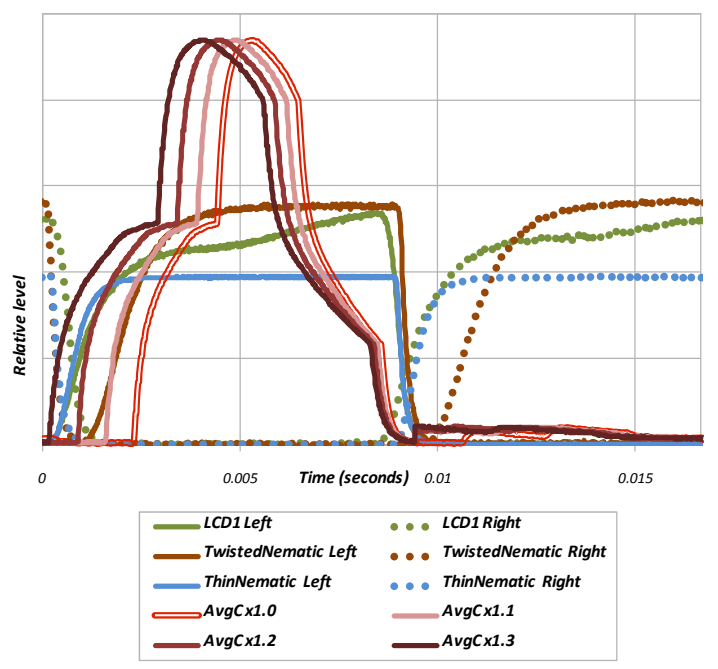

Figure 16 : Varying pulse width for LCD1 display $A v g C$ signal (scale factors $1.0,1.1,1.2,1.3$ )

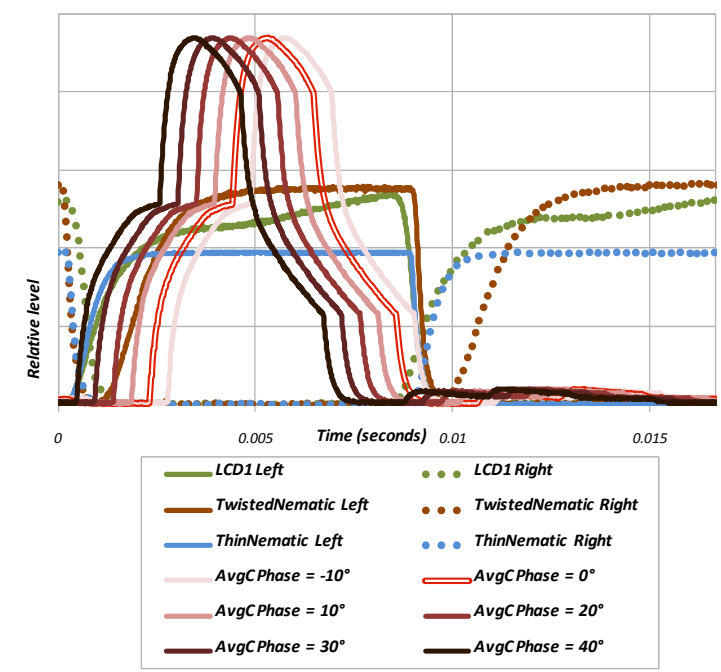

Figure 17 : Varying pulse delay for LCD1 display $\operatorname{Avg} C$ signal (phases $-10^{\circ}, 0^{\circ}, 10^{\circ}, 20^{\circ}, 30^{\circ}$, $40^{\circ}$, with $360^{\circ}=1 / 60 \mathrm{~s}$ )

\subsection{Simulation Results}

The study examined the different causes of crosstalk depending on the technology. In all cases, the crosstalk is a complex combination of several factors depending on the display panels, the shutter glasses triggering and ghost busting techniques. The study tried to discriminate the main contributions to crosstalk in each case.

A plausible cause of 3D impairments observed on commercial 3D LCD screens is the mismatch of 2D-HDTV technologies to 3D, notably concerning crosstalk. Legacy LC technologies such as $\mathrm{MVA}^{2}$ appear too slow to support the $240 \mathrm{~Hz}$ frame rate required for active glasses 3D displays. Furthermore displaying 3D content left/right disparities is much more demanding than displaying an up-converted $2 \mathrm{D}$ content. This increases requirements in terms of LC response time, especially for grey to grey transitions. Upgrading to new, faster and more efficient LC technologies in displays appears to be a major task to follow. Not only will the 3DTV signal be of higher quality, with less cross-channel display leakage, but the 3DTV system will benefit from high performance 3D glasses technologies already developed in the 3D cinema framework [5].

\footnotetext{
${ }^{2}$ MVA: Multi-domain Vertical Alignment
} 


\subsubsection{Simulation of scale and delay on LCD1 signal}

The simulation principles presented in $\S 2.2$ has been applied to the LCD1 display. Figure 18 summarizes the results.

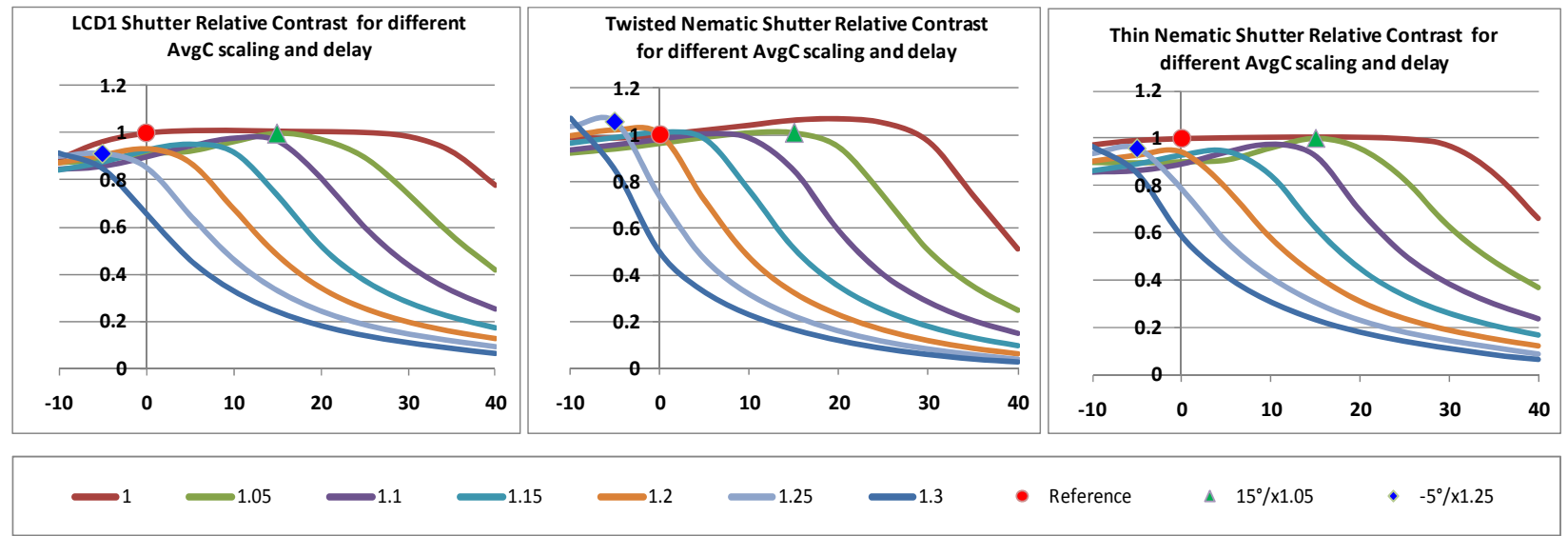

Figure 18 : Relative diaphoty photopic contrast computed for 3 different shutter technologies (LCD1 glasses, Twisted Nematic shutter and Thin Nematic shutter) varying scale and delay of the $A v g C$ signal.

$\mathrm{Y}$ axis: Relative contrast, $\mathrm{X}$ axis: Delay from $-10^{\circ}$ to $40^{\circ}$, Scale varying per curve from $\mathrm{x} 1.0$ to $\mathrm{x} 1.3$

On Figure 18 diaphoty photopic contrast is computed for the 3 different shutter technologies and for all modified versions of $A v g C$ signal. Every result is normalized by the diaphoty photopic contrast computed for the original $A v g C$ signal (delay $=0^{\circ}$, scale factor $=1.0$ taken as reference), providing a relative contrast value $\left(C_{R}\right)$ giving the possibility to evaluate improvement $\left(C_{R}>1.0\right)$ or deterioration $\left(C_{R}<1.0\right)$ due to the scaling or delay of $A v g C$ signal.

Improvement is possible for the Twisted Nematic shutter - relative contrast can be greater than the original AvgC signal case for delays between $20^{\circ}$ and $30^{\circ}$ for a scale factor 1.0 (no scaling). This may be due to the larger dark time exhibited by Twisted Nematic shutter response compared to the other two technologies (later rise). As well for the Twisted Nematic shutter, a contrast higher than the original AvgC signal is shown when a phase delay of $-5^{\circ}$ and a scaling factor of 1.25 are applied. This could seem of interest as in this case more light output could be available from the display. However this is a situation where the AvgC signal light is emitted before the Twisted Nematic shutter opens (see Figure 19) and can in practice result in a local attenuation of the bottom lines of the screen as the early signal corresponds to C5 spot measurement.

The LCD1 shutter and Thin Nematic shutter do not show a possible improvement (contrast relative to the original AvgC signal is always computed smaller than 1.0) and both express a similar behavior for the various AvgC transformed signals. Contrast is improved with Thin Nematic by adapting the birefringence. It was not the case for the shutter tested with this LCD. In contrast, optimized Thin Nematic glasses have been used for PDP experiments. The no-scaling case shows a flat curve for relative contrast variation between $0^{\circ}$ and $25^{\circ}$ phase delays. A phase delay up to $25^{\circ}$ can be applied without compromising the diaphoty photopic contrast in this case. Two positions have being identified on the graphs. Delay $15^{\circ}$ and scale $\mathrm{x} 1.05$ (green triangle) is a case where contrast is as good as in the original $\mathrm{AvgC}$ signal configuration $\left(C_{R} \approx 1.0\right)$ with a signal scaled by 1.05 so with a better light efficiency. Similarly, and this time mainly for the Thin Nematic shutter, delay $-5^{\circ}$ and scale $\mathrm{x} 1.25$ (blue diamond) is a case where contrast is as well close to the one of the original $A v g C$ signal configuration $\left(C_{R} \approx 1.0\right)$ with a signal scaled by 1.25 , so with potentially an even higher light efficiency. This time the signal expansion remains in correspondence with the two shutters open states (see Figure 19) so this could be a realistic case for improvement of light efficiency, but without crosstalk improvement actually. 


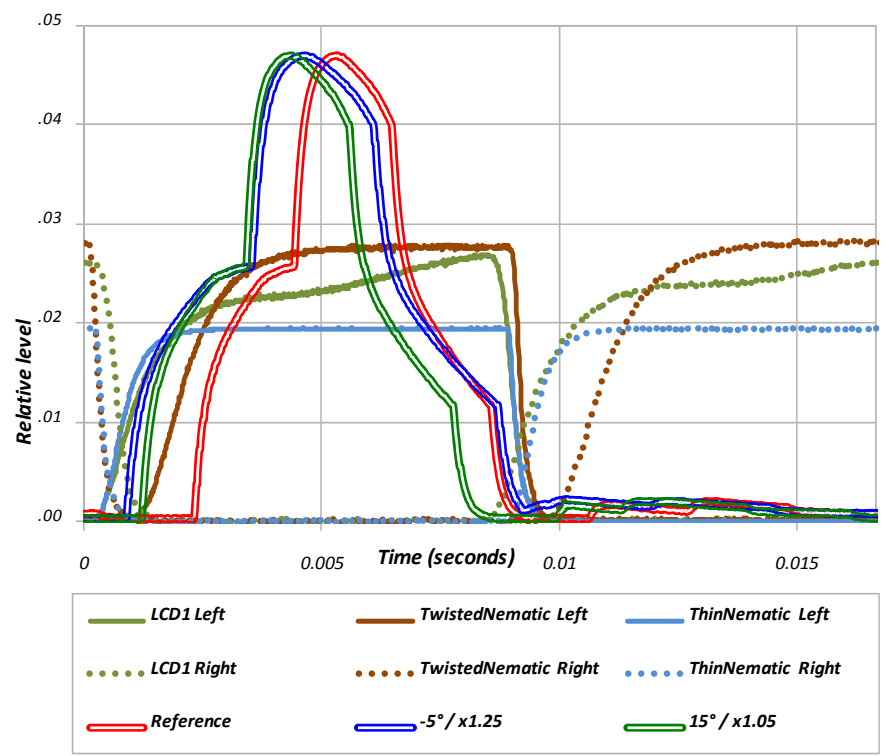

Figure 19 : Shutter responses vs. reference light signal and two specific simulated cases: $-5^{\circ} / \mathrm{x} 1.25$ and $15^{\circ} / \mathrm{x} 1.05$

For each shutter technology other measurements show that the unintended signal increases more rapidly than the intended signal when the scale factor or the delay increases. Knowing that the diaphoty photopic contrast is the ratio of the intended to the unintended signal, these measurements confirm that scaling or delaying the display pulse will not improve much the diaphoty photopic contrast. Corresponding to the discussion above, there is some reduction of the unintended signal for the Twisted Nematic shutter with scale 1.0 and delay between $10^{\circ}$ and $30^{\circ}$. This confirms that the improvement comes from less unintended light intercepted by the open shutter, presumably as having a delayed rise time compared to other shutter models.

\section{EVALUATION OF PLASMA DISPLAY PANEL (PDP) BASED 3D DISPLAYS}

PDP is an emissive display, with only two intended states (bright and dark). In the 2D-mode, for gradual grey levels reproduction, each frame is split into several sub-fields (e.g. up to 12), successively displayed with different periods, the first being usually the shorter one. In the 3D-mode, due to the phosphor lag, this order is reversed to avoid the largest subfields to still emit during the second half-period. Furthermore, in the 3D-mode the grayscale portrayal is impacted, therefore the number of sub-field per picture has been reduced. The next picture, Figure 20, presents the chronograms of a standard PDP TV. Due to the short response of the phosphor lag the crosstalk effect is less noticeable but is color dependent. For a high fidelity of color reproduction the Photo set-up was chosen as image mode. The colors temperature was set on Normal mode and a medium value was chosen for Brightness and Contrast. 

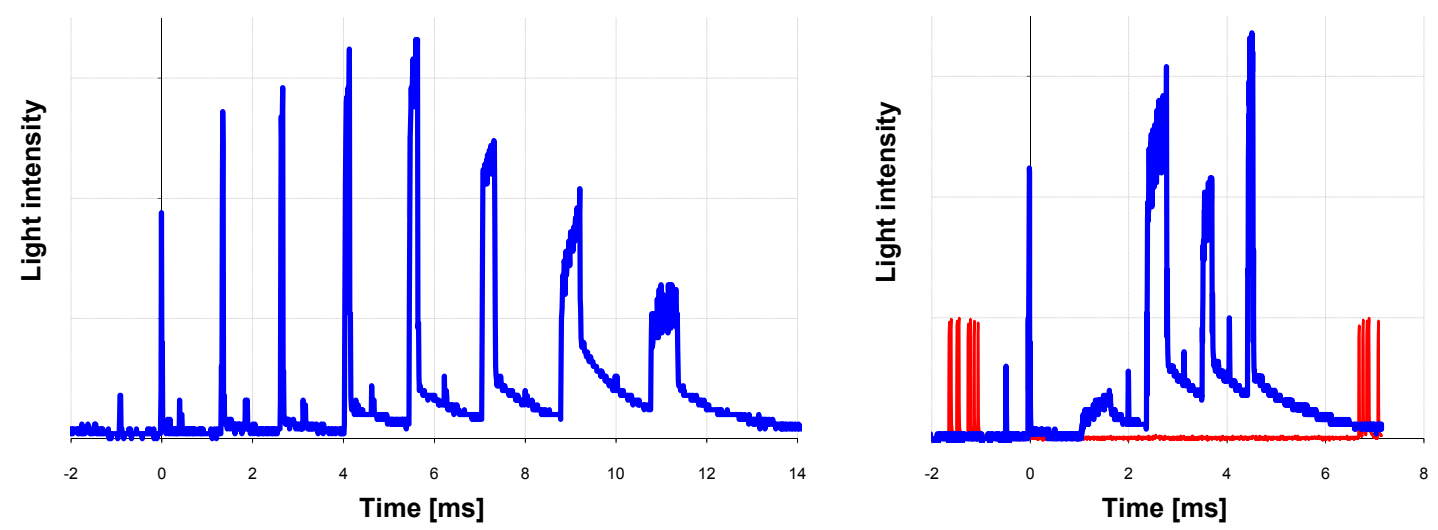

Figure 20. Chronogram of a PDP in 2D-mode (left) and 3D-mode (right). The red curve represents the IR signal emitted in $3 \mathrm{D}$-mode for electrical driving of the active glasses.

The shorter time of a frame imposes a reduction of time between the sub-fields. Furthermore, in the 3D-mode the grayscale portrayal is impacted, and the number of sub-fields is reduced. The experiments were performed on standard PDP, after testing several products, before choosing. We noticed, that in order to constantly improve the brightness at normal incidence, manufacturers preferred to reduce the vertical viewing angle. As an illustration, figure 18 shows the angular emission diagram of a standard PDP and of its most recent version, measured using the EZLite instrument and processed using EZContrast software from Eldim. The most recent PDP presents higher brightness at normal incidence $\left(1800 \mathrm{~cd} / \mathrm{m}^{2}\right.$ instead of 1400) but a vertical viewing angle highly reduced. All presented measures were performed using this more recent model.
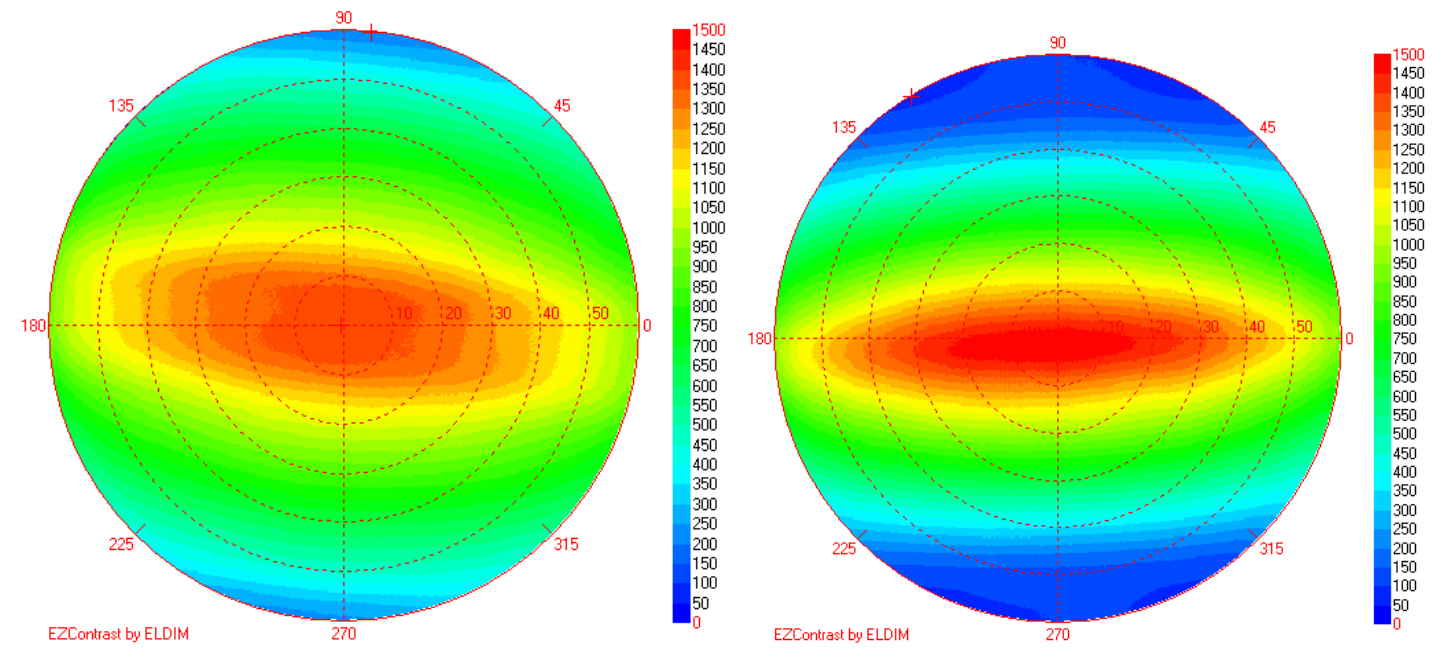

Figure 21. Viewing angles of a standard plasma display (left) and its most recent version (right).

The active glasses (mandated with a PDP) have been chosen between the commercially available and designed to operate with this plasma 3DTV panel. We compared the original glasses proposed by the TV manufacturer to some Universal glasses (here called usual TN) using the same technology (i.e. liquid crystal shutters using a Twisted Nematic (TN) texture have a large viewing angle). To be consistent with the LCD benchmark, we added a pair of glasses using Thin Nematic shutters with untwisted texture (viewing angle for Thin Nematic technology is less uniform but the response time is definitely shorter: $1.0 \mathrm{~ms}$ instead $1.8 \mathrm{~ms}$ for $\mathrm{TN}$ ). The main goal was to investigate how the different liquid crystal textures and the different response time could affect the brightness and the chrominance. The light transmission was measured for each primary color at normal incidence. Measured values are presented in the left hand side of Figure 22. 
These values are time averaged. Compared to the optical transmission measured for the open shutters, for operating glasses the transmission varies from 46 to $48 \%$ of the transmission of open shutters for all technologies.
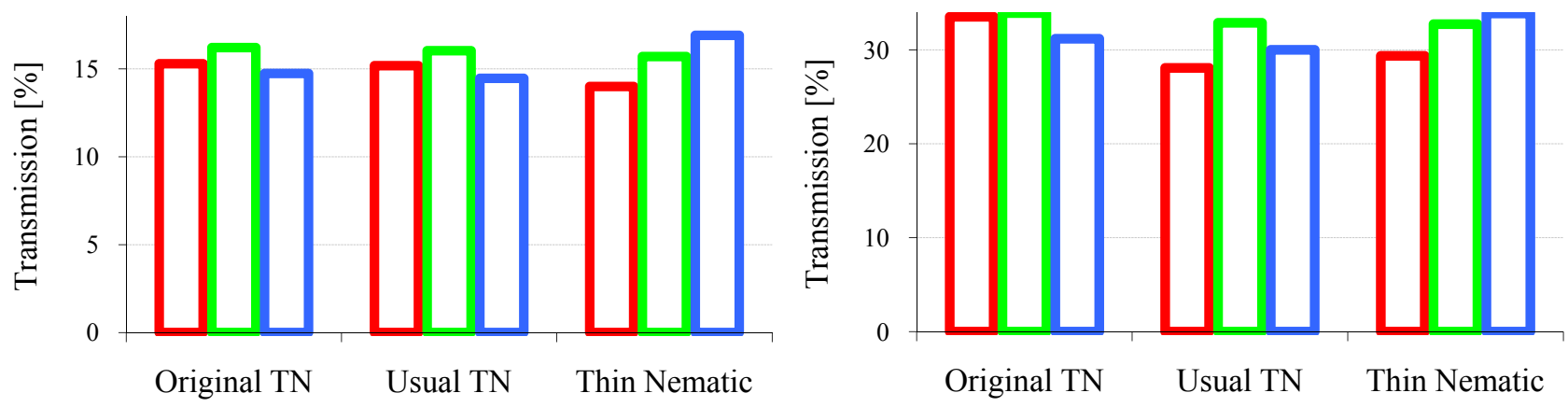

Figure 22. Measured light transmission for the 3 primary colors (Red, Green and Blue). At the left for the operating glasses, at the right side the static measurements.

Taking into account the different levels of primary colors $\left(954 \mathrm{~cd} / \mathrm{m}^{2}\right.$ for Red, $2700 \mathrm{~cd} / \mathrm{m}^{2}$ for Green and $418 \mathrm{~cd} / \mathrm{m}^{2}$ for Blue), obviously different values of transmission impact the color balance. Figure 20 shows how the colors of a uniform white screen change when looking through the glasses at normal incidence.

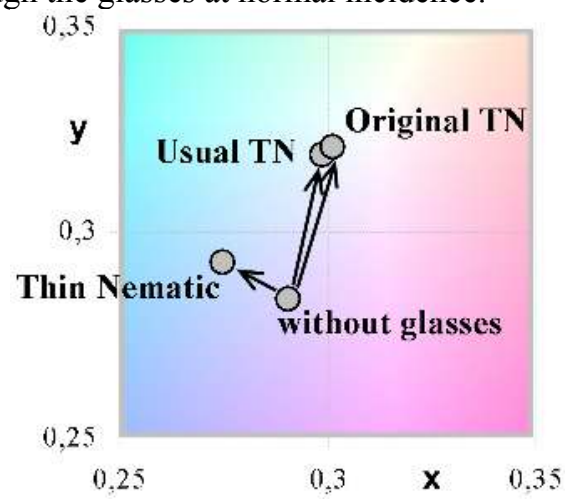

Figure 23. Color changes for a white image displayed on PDP. The colors are characterized by the $\mathrm{x}$ and $\mathrm{y}$ coordinates of the CIE 1931 color space [7].

As we can observe, all 3D glasses introduce hue changes. The TN a little more, but the manufacturer clearly adapted the white point of the screen, to obtain, when viewing through the glasses, a better white. The color changes produced by the Thin Nematic shutters are weaker and can be compensated similarly by image pre-processing. Nevertheless, this compensation could be possible only if the hue changes are uniform for all viewing directions. For this reason we investigated the color changes for tilted viewings. Figure 24shows the color aspect of the screen through the 3D active glasses, when a white image is displayed on the PDP. 


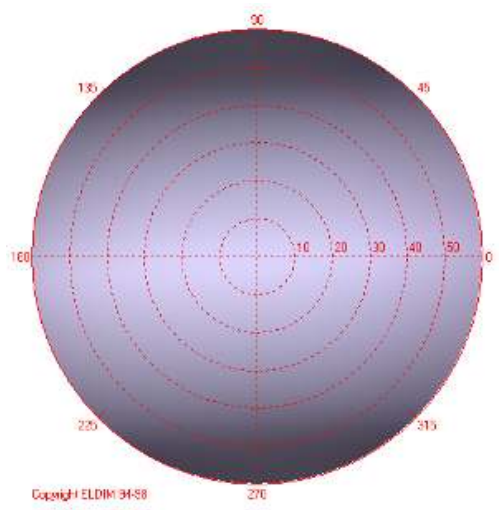

a). Without glasses

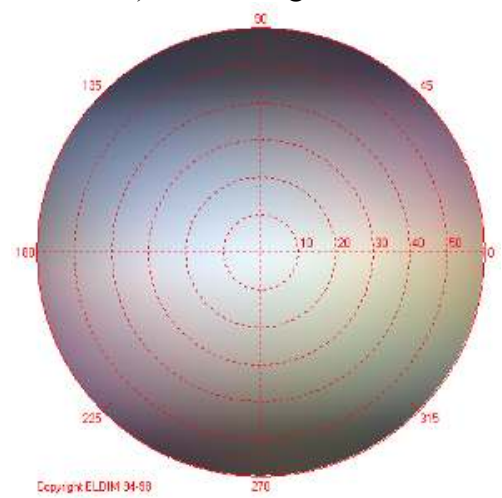

c). Usual TN shutters

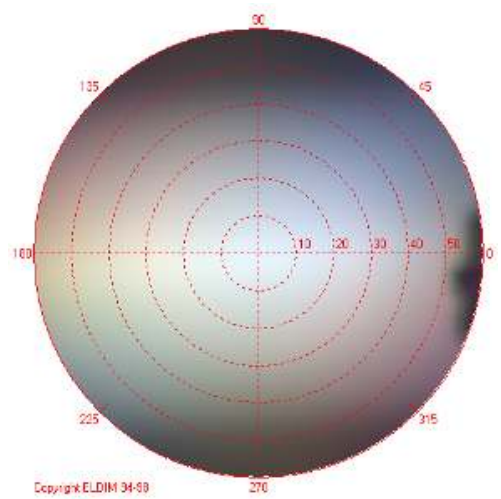

b). Original TN glasses

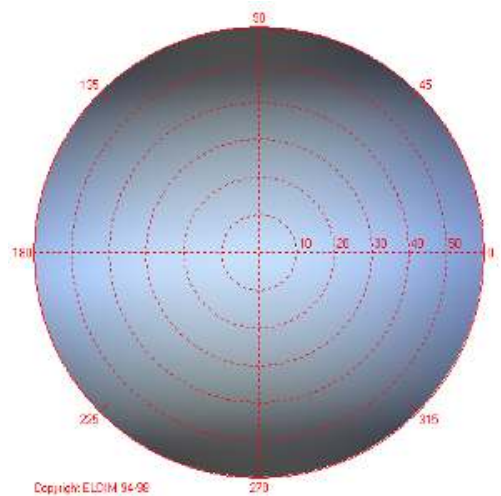

d). Thin Nematic shutters

Figure 24. Angular dependence of the color for a white screen viewed without glasses (a.) and with the glasses using Twisted Nematic (b. and c.) or Thin Nematic (d.) shutters

The first result shows a lack of uniformity of the color changes for both TN shutters. Unfortunately, this is a serious drawback which cannot be compensated by image pre-or post-processing. For the Thin Nematic technology, the shutters exhibit a more uniform color modification. This fact becomes obvious when we compare (Figure 25) the color dispersion of the white and the three primary colors for the different $3 \mathrm{D}$ glasses.

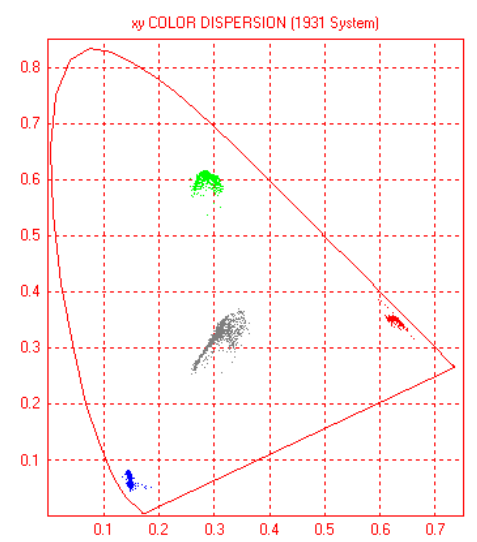

a). Original $\mathrm{TN}$ glasses

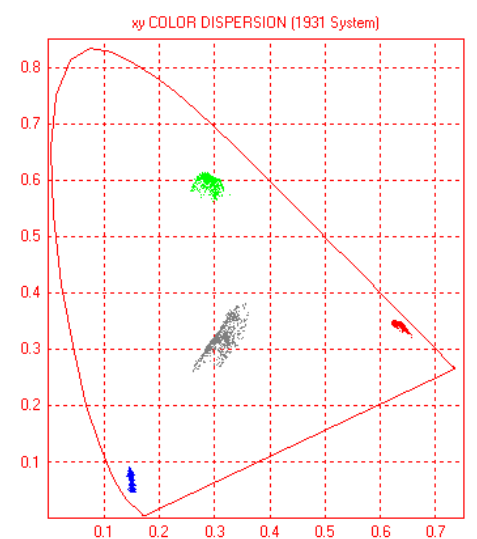

b). Usual TN shutters

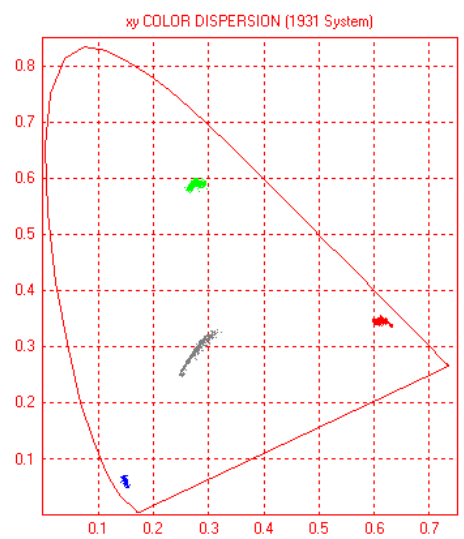

c). Thin Nematic shutters

Figure 25. Color dispersion of the White (grey dots), Red, Green and Blue screens viewed through the 3D glasses: Original TN (a.), Usual TN (b.) and Thin Nematic (c.) 
The last measurement concerns the angular dependence of the optical transmission. Both technologies give similar results at normal incidence. However, the angular dependence is different (see Figure 26).

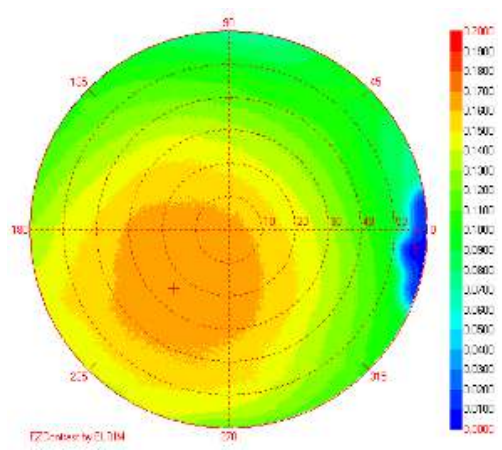

Original TN glasses

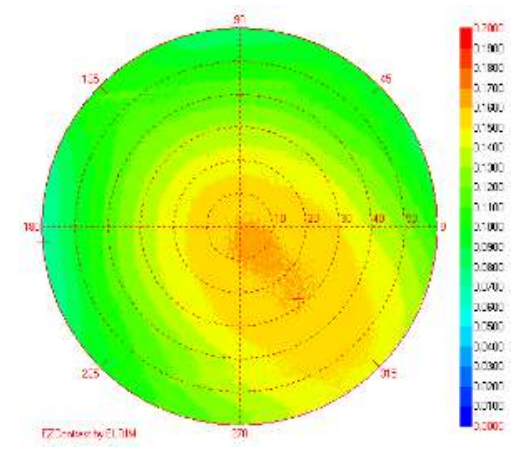

Usual TN shutters

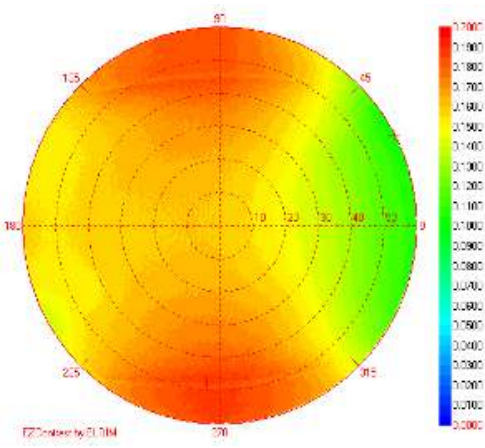

Thin Nematic shutters

Figure 26. Angular dependence of the optical transmission for a white screen for operating glasses: Original TN glasses (a.), Usual TN (b.) and Thin Nematic (c.)

For Twisted Nematics we observe the typical shape of a TN with a preferential viewing direction at $45^{\circ}$ beside the polarizers. For Thin Nematic the viewing angle is symmetric and obviously the manufacturer used this intrinsic property to compensate the viewing angle of the PDP. To conclude, as previously we notice that switching times have a little impact on the average transmission. This fact can be explained, if we consider the large dark-time between the frames (almost 2ms) which has been chosen extremely large (despite a luminance penalty), to prevent from crosstalk, therefore, Thin Nematic could be used efficiently to reduce this dark time. Both technologies (Twisted Nematic and Thin Nematic) introduce color changes. However, color dispersion is lower an more uniform for Thin Nematic shutters, making easier color compensation, by image pre-processing. In addition, the angular symmetry of the optical transmission for the Thin Nematic shutter could be used to improve (or compensate) the limited zenithal viewing angle of the PDP.

\section{CONCLUSIONS}

We studied crosstalk on LCD and PDP with the objective to identify the source of artifacts, with the help of physical measurements and simulations. Screen and shutter glasses contributions have been evaluated to figure out possible improvements. Concluding definitively from the presented work is difficult as based on a non-exhaustive display benchmark, and as technologies are in permanent evolution. However, from our study, it arises that, for LCD screens, according to the current state of what is commercially available, improvements will mainly result from LCD panel manufacturers, seeking the reduction of cross-channel display leakage involving higher performance LC technologies and corresponding backlight strategies. Simultaneously, optimized shutter glasses can bring their valuable contribution to the overall quality improvement when included in the full viewing chain optimization. For Plasma displays the use of more efficient shutter glasses (faster) can improve the color fidelity, by increasing the number of sub-fields and reducing the risks of color banding and in parallel compensating the viewing angle particularly in the vertical direction (we have seen in the PDP case that glasses can be used for that purpose). In the current state of the art, most of manufacturers proposed to improve 3D displays quality with respect to crosstalk by using ghost busting techniques, usually based on subtracting light on one channel image, where a leakage can happen from the other channel [8]. However, some crosstalk aspects cannot be compensated by such a technique (e.g. one cannot remove light from a dark/black area). Moreover, ghosting compensation techniques do not appear to be perfect where they could be in theory, due to the complexity of the crosstalk phenomenon that has to be modeled in the ghost busting process. Undoubtedly upgrading technologies in their physics (improvement of response times) and in the reduction of cross-channel display leakage would be a sounder approach than only ghost busting images. 


\section{REFERENCES}

[1] Woods, A. and Tan, S. (2002). "Characterising sources of ghosting in time sequential stereoscopic video displays". Proceedings of the SPIE, 4660:66-77.

[2] Janusz Konrad J., Lacotte B., Dubois E., "Cancellation of image crosstalk in time-sequential displays of stereoscopic video", IEEE Transactions on Image Processing, Vol. 9, No. 5, pp. 897-908, May 2000

[3] Boher P., Leroux T., Collomb-Patton V., "Characterization of one Time-Sequential Stereoscopic 3D Display Part I: Temporal Analysis", JSID, Vol 11, N², 57 (2010)

[4] Sacré J. J., et al. "Diversity and coherence of 3D Crosstalk Measurement". SID conferences, Los Angeles, CA, May 2011. SID Symposium Digest 42, 804 (2011)

[5] Srivastava K., et al., "Liquid Crystal Active Glasses for 3D Cinema", Journal of Display Technology, Vol. 6, $\mathrm{N}^{\circ}$. 10, Oct. 2010, pp 522-530.

[6] Deschamps J., Doyeux H., "Plasma displays," in Physics World, Vol. 10, pp. 39-43, 1997

[7] Malacara D., "Colour Vision and Colorimetry: Theory and Applications", SPIE Press Monograph, 2002, Vol. PM105.

[8] Lipscomb J. S., Wooten W. L., "Reducing crosstalk between stereoscopic views". SPIE/SD\&A V, published in Proceedings of SPIE Stereoscopic Displays and Virtual Reality Systems, vol. 2177, pp. 92-96, Feb. 2004. 